\title{
Radiation Absorption Characteristics of Titanium Alloys
}

\author{
S.J. Stankovic*, R.D. Ilic, D.M. Davidovic, M. Petrovic, S. Tadic and M. Kovacevic \\ Vinca Institute of Nuclear Science, Radiation and Environmental Protection Laboratory \\ P.O. Box 522, 11001 Belgrade, Serbia
}

\begin{abstract}
Titanium alloys have found numerous applications in space research, and nuclear industry and research. Since X-rays constitute an important part of the space radiation environment, numerical simulations of radiation absorption characteristics of titanium alloys were studied in this paper. The photon transport Monte Carlo software was used for determining the energy deposited in titanium samples. The numerical results show the pronounced dependence of radiation absorption properties of different combinations of components in alloy. The results obtained are encouraging in respect of optimization of structure of alloys regarding their required features in radiation shielding.
\end{abstract}

PACS numbers: 79.20.Ap

\section{Introduction}

The development of modern nuclear sciences and practice rooted in nuclear power engineering and further advancement of the space exploration are determined to a large extent by inventions of metallic materials intended for various functional applications. These applications require reliable service, economic efficiency and minimum disruption of ecology. The successful realization of these requirements depends mainly on the application of metals and alloys characterized by high radiation resistance and accelerated decrease in induced radioactivity [1]. The successful implementation of the aluminides depends on ability to extend their application spectrum, and thus reduce scale-related costs. For this to happen, processability to a range of milli forms and products will be established, especially for TiAl alloys [2]. For example, the detection method of real time radioscopy was adopted to test titanium alloys precision weldments with complex structure non-destructively. The location of small defects in the gray distribution curve was determined analyzing the X-ray detection images and geometry relationship of precision weldments components, and a formula allowing the calculation of the depth of defects was deduced [3].

Titanium alloy (Ti-13Nb-13Zr) parts are ideally suited for advanced aerospace systems because of their unique combination of high specific strength at both room temperature and moderately elevated temperature, in addition to excellent corrosion resistance. Despite these features, use of titanium alloys in engines and airframes is limited by cost [4]. To better predict

* corresponding author; e-mail: srbas@vinca.rs radiation-induced segregation in alloys, a model is developed which uses pair interaction energies, ordering energies and local atomic configuration to calculate diffusion parameters [5]. Since X-rays constitute an important part of the space radiation environment, numerical simulations of radiation absorption characteristics of titanium alloys were studied in this paper.

\section{Theory}

When an atom of a metal (alloy) receives from a moving particle an energy equal to or higher than some threshold value $E_{\mathrm{T}}$ (of the order $20-40 \mathrm{eV}$ ), the atom is displaced from its position in crystal lattice. Depending on the energy of the primary knocked out atom $E_{\mathrm{K}}$ which is mainly determined by the type and energy of incident radiation, the nature of irradiated target, the crystallographic direction and the angle of collision of the particle with atom of the lattice, various configurations and structures of radiation defects may appear, starting from isolated point defect-vacancies and interstitial (the Frenkel pairs) and ending with spatial zones of damage characterized by a complicated structure of defects. The defects, formed during elementary acts of the interaction of the moving particles with matter usually belong to the primary radiation defects. The subsequent kinetic evolution of the structure of radiation damage is determined by the processes of spatial overlapping of the volumes of primary damage and/or diffusion of freely-migrating point defects and solutes.

The actual theoretical analysis of the formation of primary radiation defects is carried out using two main approaches: calculations using theory of simple binary collisions and the modified Kinchin-Pease model, and com- 
puter modeling using the molecular dynamics method.

Within the framework of the theory of binary collisions, the total number of the Frenkel pairs, formed during the transfer of energy $E_{\mathrm{K}}$ to a primary-displaced atom from the particle with the initial energy $E_{\mathrm{IN}}$, is given by the equation [1]:

$$
F_{\mathrm{P}}\left(E_{\mathrm{IN}}\right)=\int_{E_{\mathrm{T}}}^{E_{\mathrm{MAx}}} \mathrm{d} E_{\mathrm{K}} \frac{\mathrm{d} \sigma\left(E_{\mathrm{IN}}, E_{\mathrm{K}}\right)}{\mathrm{d} E_{\mathrm{K}}} F_{\mathrm{P}}\left(E_{\mathrm{K}}\right) .
$$

In Eq. (1) $\mathrm{d} \sigma\left(E_{\mathrm{IN}}, E_{\mathrm{K}}\right) / \mathrm{d} E_{\mathrm{K}}$ is the differential cross-section for the transfer by an incident particle of energy in the range from $E_{\mathrm{K}}$ to $E_{\mathrm{K}}+\mathrm{d} E_{\mathrm{K}}$ to the primary-displaced atom, $F_{\mathrm{P}}\left(E_{\mathrm{K}}\right)$ is the number of the Frenkel pairs formed by recoil atoms of this energy range, $E_{\mathrm{T}}$ is the threshold energy of displacement, and $E_{\mathrm{MAX}}$ is the maximum energy which can be transferred by the particle to the primary-displaced atom.

The maximum energy $E_{\mathrm{MAX}}$ is the function of deposited energy per material density and thickness (or volume) of specimen $\left(\mathrm{d} E_{\mathrm{D}} / \rho \mathrm{d} z\right)$ in $\mathrm{MeV} \mathrm{cm} / \mathrm{g}$ in the $z$-axis direction. In this way, radiation absorption characteristics of alloys are defined via macroscopic quantity as deposited energy in alloy specimen.

\section{Numerical method}

\subsection{Materials and geometry}

In our numerical experiments, we defined geometry of specimen as parallelepiped $2 \times 2 \times 2 \mathrm{~cm}^{3}$. Materials of specimens were titanium alloys: $\mathrm{Ti}-24 \mathrm{Al}-11 \mathrm{Nb}$ with $\mathrm{Ti}(64 \%), \mathrm{Al}(24 \%), \mathrm{Nb}(11 \%), \mathrm{V}(1 \%)$ and $\mathrm{Ti}-13 \mathrm{Nb}-$ $13 \mathrm{Zr}$ with $\operatorname{Ti}(62.65 \%), \mathrm{Nb}(22.09 \%), \operatorname{Zr}(15.26 \%)$. The appropriate geometry form of the alloy specimen was defined in accordance with corresponding software program [6]. FOTELP-2K6 program uses Research Focus Group (RFG) geometry based on Rvachev R-functions, for the specimen geometry description.

\subsection{Monte Carlo code}

The calculation of deposited energy for photon transport through alloy specimen was realized by FOTELP$-2 \mathrm{~K} 6$ code [6]. FOTELP-2K6 is developed for numerical experiments by Monte Carlo techniques for dosimetry, radiation damage, radiation therapy and other actual applications of these particles. For the photon history, following it from scattering to scattering using corresponding inverse distribution between collision, types of target, types of collisions, types of secondary, their energy and scattering angles generates the trajectory. Photon interactions are coherent scattering, incoherent scattering, photoelectric absorption and pair production. The Doppler broadening in the Compton scattering is taken. The histories of secondary photons include bremsstrahlung and positron-electron annihilation radiation. The condensed history Monte Carlo method is used for the electron and positron transport simulation.

During a history the particles lose energy in collisions, and the secondary particles are generated on the step according to the probabilities for their occurrence. Electron (positron) energy loss is through inelastic electronelectron $\left(\mathrm{e}^{-}, \mathrm{e}^{-}\right)$and positron-electron $\left(\mathrm{e}^{+}, \mathrm{e}^{-}\right)$collisions and bremsstrahlung generation. The fluctuation of energy loss (straggling) is included according to the Landau or Blunk-Westphal distributions with 9 Gaussians. The secondary electrons, which follow history of particles, include knock-on, pair production, Compton and photoelectric electrons. The secondary positrons, which follow pair production, are included, too. With atomic data, the electron and positron Monte Carlo simulation is broadened to treat atomic ion relaxation after photoeffect and impact ionization. Physical rigor is maximized by employing the best available cross-sections and high speed routines for random values sampling from their distributions, and the most complete physical model for describing the transport and production of the photon/electron/positron cascade from $100.0 \mathrm{MeV}$ down to $1.0 \mathrm{keV}$.

\section{Results and discussion}

In numerical experiments, photon beam was positioned in the direction that is perpendicular to the front surface of alloy specimen. Deposited energy as radiation absorption characteristic was obtained by the Monte Carlo simulations with ${ }^{60} \mathrm{Co}$ photon incident beams with average energy of $1.25 \mathrm{MeV}$. Simulations were performed with 1 million photon transport histories.

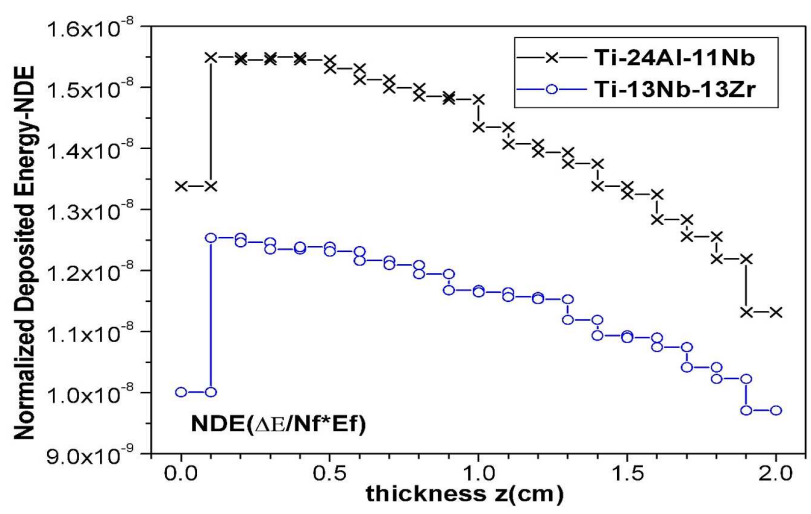

Fig. 1. Normalized deposited energy vs. thickness of alloy specimens. $N_{f}=10^{6}, E_{f}=E_{\mathrm{IN}}=1.25 \mathrm{MeV}$.

Deposited photon energies in the material zones of two Ti-alloys are shown in Fig. 1. Radiation impact for alloy of $\mathrm{Ti}-24 \mathrm{Al}-11 \mathrm{Nb}$ is greater than for alloy of $\mathrm{Ti}-13 \mathrm{Nb}-$ 13Zr. The total number of the Frenkel pairs, formed during transfer of energy $E_{\mathrm{K}}$ to a primary-displaced atom from a particle with the initial energy $E_{\mathrm{IN}}$ is greater for alloy with aluminum than for alloy with zirconium.

\section{Conclusion}

The present work, numerical experiments using FOTELP-2K6 code were performed in order to deter- 
mine radiation absorption characteristics of titanium alloys. The numerical results show the pronounced dependence of radiation absorption properties as a function of different combinations of components in alloy. The results obtained are encouraging because they show the possibilities and advantages of use of such an approach in optimization of structure of alloys with respect to required features in radiation shielding.

\section{References}

[1] L.I. Ivanov, Yu.M. Platov, Radiation Physics of Metals and its Applications, Cambridge International Science Publishing, Cambridge (UK) 2004.

[2] D. Banerjee, MRS Bull. 26, 223 (2001).
[3] T. Gang, D.H. Shi, Y. Yuan, S.Y. Yang, J. Phys., Conf. Series 48, 1341 (2006).

[4] V.A.R. Henriques, P.P. de Campos, C.A.A. Cairo, J.C. Bressiani, Mater. Res. 8, 443 (2005).

[5] T.R. Allen, G.S. Was, Acta Mater. 46, 3679 (1998).

[6] R.D. Ilić, FOTELP-2K6, Photons, Electrons and Positrons Transport in $3 D$ by Monte Carlo Techniques, IAEA1388, FOTELP-2K6, http://www.nea.fr/abs/html/iaea1388.html . 